https://doi.org/10.15407/ujpe64.6.487

A.V. KHOMENKO

Sumy State University

(2, Rimskii-Korsakov Str., Sumy 40007, Ukraine; e-mail: o.khomenko@mss.sumdu.edu.ua)

\title{
SELF-SIMILAR MODE OF METALS FRAGMENTATION UNDER SEVERE PLASTIC DEFORMATION
}

\begin{abstract}
In the framework of nonequilibrium evolution thermodynamics, the influence of additive fluctuations on the kinetics of structural defects under severe plastic deformation has been studied. The applied method is a new one for the description of fragmentation modes and corresponding self-organization processes. It is found that a fragmented metallic specimen demonstrates a self-similar behavior, which results in the formation of a grain structure with various grain sizes. Such a behavior takes place provided that the probability distribution for the grain boundary density has a power-law dependence. A comparison of the results obtained in the Itô and Stratonovich forms demonstrates the absence of qualitative changes in the behavior of the system.

Keywords: grain boundary, dislocation, phase transition, phase diagram, internal energy, additive noise, self-similarity.
\end{abstract}

\section{Introduction}

It is known that, during the fragmentation of a metal specimen, which is performed with the help of severe plastic deformation (SPD) methods and under certain conditions, the so-called fractal structures are formed in the material [1-8]. As a result, the emerged grain (stationary) structure of the metal is characterized by the power-law size distribution for the ensemble of grains with various sizes. The characteristic scale of those grains cannot be determined (in our case, we are interested in the scale for the density of grain boundaries $\left.(\mathrm{GBs}), h_{g}\right)$. When the specimen surface increases (the growth factor can be arbitrary in this case), the system demonstrates a selfsimilar behavior, i.e. the surface morphology always remains similar to itself. In other words, the system preserves relationships between its state parameters $[9,10]$.

To describe the process of metal structure fragmentation, a generalized thermodynamic model was developed in works [11-18]. This model combines the methods of the classical non-equilibrium thermodynamics and the Landau theory of phase transitions. It allowed one to describe the kinetics of the defect subsystem (dislocations and GBs) and to analyze

(C) A.V. KHOMENKO, 2019

ISSN 2071-0194. Ukr. J. Phys. 2019. Vol. 64, No. 6 both the formation conditions and the stability of the formed submicrocrystalline (SMC) or nanocrystalline (NC) boundary (stationary) structures. At the same time, the proposed theory considers the influence of fluctuations of the main parameters (noise), which can substantially change the evolution of the system in some cases and even lead to the appearance of new states $[16,19-22]$ that cannot be realized in the deterministic case [11-14, 23-25].

In this work, it was shown that the introduction of additive noise for the main parameters makes it possible to describe the self-similar behavior of structural defects during the formation of SMC or NC boundary structures. The conditions for the formation of quasifractal granular structures were analyzed. It was found that the grain-size distribution function acquires a power-law form, if the GB density parameter lies within the interval $10^{-5} \mathrm{~m}^{-1} \lesssim h_{g} \lesssim 10^{10} \mathrm{~m}^{-1}$. A comparison of the results obtained showed that the choice of the Itô and Stratonovich calculus affects only the dispersion of fluctuations of the state parameter $h_{g}$ around the stationary values of the system and gives rise to the renormalization of the spectral distribution of grain sizes in the formed NC or SMC boundary structure. At the same time, the actual stationary values of the GB density do not depend on the interpretation choice. 


\section{Effective Potential}

Let us consider the basic potential for the internal energy density in the form $[11,19]$ :

$u\left(h_{g}, h_{D}\right)=u_{0}+\sum_{m=g, D}\left(\varphi_{0 m} h_{m}-\frac{1}{2} \varphi_{1 m} h_{m}^{2}+\right.$
$\left.+\frac{1}{3} \varphi_{2 m} h_{m}^{3}-\frac{1}{4} \varphi_{3 m} h_{m}^{4}\right)+\varphi_{g D} h_{g} h_{D}-\psi_{g D} h_{g}^{2} h_{D}$,

where

$u_{0}=\frac{1}{2} M\left(\varepsilon_{i i}^{e}\right)^{2}+2 \mu I_{2}$,

$\varphi_{0 m}=\varphi_{0 m}^{*}+g_{m} \varepsilon_{i i}^{e}+\left(\frac{1}{2} \bar{M}_{m}\left(\varepsilon_{i i}^{e}\right)^{2}+2 \bar{\mu}_{m} I_{2}\right)$,

$\varphi_{1 m}=\varphi_{1 m}^{*}+2 e_{m} \varepsilon_{i i}^{e}$.

In formulas (1)-(4), $u_{0}$ is a part of the internal energy that does not depend on the imperfection in the material structure (the reference level); $h_{g}$ and $h_{D}$ are the GB and dislocation densities, respectively ${ }^{1}$; the subscript values $m=g$ and $m=D$ denote the GBs and dislocations, respectively; $M=\lambda+2 \mu$ is the modulus of uniaxial compression of a material $[26,27] ; \lambda$ and $\mu$ are the Lamé constants; $\varepsilon_{i i}^{e}$ and $I_{2} \equiv\left(-\varepsilon_{i i}^{e} \varepsilon_{j j}^{e}+\varepsilon_{i j}^{e} \varepsilon_{j i}^{e}\right) / 2$ are the first and second, respectively, invariants of the elastic strain tensor; $\varphi_{0 m}^{*}$ is the characteristic defect energy involving the defect dimensionality (per unit length for dislocations and per unit surface area for GBs); $\varphi_{0 m}$ is an analog of $\varphi_{0 m}^{*}$ taking the influence of elastic deformations in the linear (the constant $g_{m}$ ) and quadratic approximations into account; the positive constant $g_{m}$ is responsible for either the generation of structural defects at stretching $\left(\varepsilon_{i i}^{e}>0\right)$ or their annihilation at compression $\left(\varepsilon_{i i}^{e}<0\right) ; \bar{M}_{m}$ and $\bar{\mu}_{m}$ are elastic constants that reflect the decrease of corresponding elastic moduli owing to the presence of structural defects; $\varphi_{1 m}$ and $\varphi_{1 m}^{*}$ are coefficients that are responsible for the recrystallization (defect annihilation) considering and not considering, respectively, the influence of an elastic deformation in the linear approximation (constant $\left.e_{m}\right)$; accordingly, the parameter $e_{m}$ characterizes the enhancement of the annihilation process at $\varepsilon_{i i}^{e}>0$ (in the case $\varepsilon_{i i}^{e}<0$, the backward process is implied); and $\varphi_{g D}$ is a parameter that characterizes the interaction energy of selected structural defects. In the general case, the positive terms in relation (1) are responsible for the generation of structural defects, and the

\footnotetext{
1 The approximate value of the grain size $d$ is assumed to be inversely proportional to $h_{g}$, i.e. $d \sim 1 / h_{g}$.
}

negative terms correspond to the inverse processes, the defect annihilation (recrystallization).

Expression (1) for the internal energy density differs from the initial one [11-14] by the presence of the last term, which describes the self-consistent behavior of the GB density $h_{g}$ and the dislocation density $h_{D}$. According to it, the additive noise transforms into the multiplicative one [19]. The minus sign in front of this term ensures the formation of stationary states (the maximum of the thermodynamic or synergetic potential) and reflects the Le Chatelier principle. According to the latter, the thermodynamic process of a higher rank aims at compensating effects from the thermodynamic processes of lower ranks. It should be noted that such treatment methods are considered, at which the temperature at the contacting surfaces of the specimen can increase by $50-70^{\circ} \mathrm{C}$, but the melting does not occur.

The series expansion of Eq. (1), provided that the coefficients $\varphi_{k m}(k=0 \div 3)$ are positive, may result in the formation of two maxima. In the GB case, these maxima correspond to the appearance of a twomode distribution over the grain size. In the dislocation case, the mode corresponding to the smaller imperfection value describes a stochastic (uniform) distribution of the presented defect; accordingly, a larger imperfection value corresponds to an ensemble of dislocations that form a cell structure. Note that, in order to describe the formation of a boundary (stationary) structure, a higher order of approximation of the internal energy (1) in the GB density is required. But in the case of dislocations, it is sufficient to restrict the series expansion to the second power of the defect density $\left(\varphi_{2 \mathrm{D}}=0 \mathrm{~J} \mathrm{~m}^{3}\right.$ and $\left.\varphi_{3 D}=0 \mathrm{~J} \mathrm{~m}^{5}\right)$.

For the further consideration, the coefficients were assigned with the values that are quoted in Table 1 . Those parameters were partially substantiated in works $[11,12]$. It should be noted that the values for the main coefficients were obtained experimentally while studying the copper structure. Nevertheless, the proposed model (1) has a wider scope of applications. If necessary and provided that the proper parameter values are used, it will produce good results for any metal.

\section{Langevin and Fokker-Planck Equations}

Since the Gaussian white noise is one of the simplest mathematical models that is often used to de- 
Table 1. Parameters of the two-defect model with noise

\begin{tabular}{|}
\begin{tabular}{|c|c|c|c|c|c|c|c|c|}
\hline$M, \mathrm{~Pa}$ & $\varphi_{0 g}^{*}, \mathrm{~J} \cdot \mathrm{m}^{-2}$ & $g_{g}, \mathrm{~J} \cdot \mathrm{m}^{-2}$ & $\bar{M}_{g}, \mathrm{~J} \cdot \mathrm{m}^{-2}$ & $\bar{\mu}_{g}, \mathrm{~J} \cdot \mathrm{m}^{-2}$ & $\varphi_{1 g}^{*}, \mathrm{~J} \cdot \mathrm{m}^{-1}$ & $e_{g}, \mathrm{~J} \cdot \mathrm{m}^{-1}$ & $\varphi_{2 g}, \mathrm{~J}$ & $\varphi_{3 g}, \mathrm{~J} \cdot \mathrm{m}$ \\
\hline $2.08 \cdot 10^{10}$ & 0.4 & 12 & $2.5 \times 10^{5}$ & $3 \times 10^{5}$ & $3 \times 10^{-6}$ & $3.6 \times 10^{-4}$ & $5.6 \times 10^{-13}$ & $3 \times 10^{-20}$ \\
\hline$\mu, \mathrm{Pa}$ & $\varphi_{0 D}^{*}, \mathrm{~J} \cdot \mathrm{m}^{-1}$ & $g_{D}, \mathrm{~J} \cdot \mathrm{m}^{-1}$ & $\bar{M}_{D}, \mathrm{~J} \cdot \mathrm{m}^{-1}$ & $\bar{\mu}_{D}, \mathrm{~J} \cdot \mathrm{m}^{-1}$ & $\varphi_{1 \mathrm{D}}^{*} \mathrm{~J} \cdot \mathrm{m}$ & $e_{D}, \mathrm{~J} \cdot \mathrm{m}$ & $\varphi_{g D}, \mathrm{~J}$ & $\psi_{g D}, \mathrm{~J} \cdot \mathrm{m}$ \\
\hline $2.08 \times 10^{10}$ & $5 \times 10^{-9}$ & $2 \times 10^{-8}$ & 0 & $1.65 \times 10^{-4}$ & $10^{-24}$ & $6 \times 10^{-23}$ & $10^{-16}$ & $10^{-23}$ \\
\hline
\end{tabular}
\end{tabular}

scribe physical processes, let us analyze the influence of additive noises on the formation of SMC or NC boundary structures. The relevant system of kinetic equations for the order parameters looks as follows: $[13,14,19]$ :

$\tau_{h_{D}} \dot{h}_{D}=\varphi_{0 D}-\varphi_{1 \mathrm{D}} h_{D}+\varphi_{g D} h_{g}-\psi_{g D} h_{g}^{2}+\sqrt{N_{D}} \xi_{D}$

$\tau_{h_{g}} \dot{h}_{g}=\varphi_{0 g}-\varphi_{1 g} h_{g}+\varphi_{2 g} h_{g}^{2}-\varphi_{3 g} h_{g}^{3}+\varphi_{g D} h_{D}-$

$-2 \psi_{g D} h_{g} h_{D}+\sqrt{N_{g}} \xi_{g}$,

where $\tau_{h_{m}}$ are the reciprocal kinetic coefficients corresponding to the relaxation times of the dislocation and GB densities. The stochastic sources describe the fluctuations of main parameters (internal noise) with the intensities $N_{D, g}$ [28]; namely, these are various heterogeneities (substance phases, impurities, inclusion, vacancies, structural defects of other levels, thermal fluctuations, and so forth), as well as changes in the external thermostat, in particular, the defects of the experimental installation. As is known, SPD governs the formation of GBs of two types. The first ones are large-angle, or geometrically necessary, boundaries, which arise as a result of various activities of the sliding system around the GBs. The second ones are the boundaries or subboundaries of cells, which are often called random dislocation boundaries, because they arise at the mutual implementation of a statically random dislocation intersection inside the grains $[27,29]$. The boundaries between arbitrarily arranged grains are much more mobile than the latter. Grain boundaries possess a higher non-equilibrium energy, because they accumulate structural defects of other levels during the treatment, which leads to the activation of relaxation processes by invoking a plastic flow. In the course of deformation, owing to the accumulation of dislocations, the cells gradually transform into subgrains, which are confined by small-angle boundaries, but become high-angle nanograins in the future. In the case of the simplest kind of interactions between defects belonging to the same level, the emergence of fluctuations of internal variables takes place owing to the self-organization processes [20-22].

Let us introduce the functions $\xi_{i}(t)$, where $i=D$ or $g$, which represent random Gaussian quantities (white noise). They have an autocorrelation function that is mathematically described with the help of the Dirac $\delta$-function and satisfies the equalities [30]

$\left\langle\xi_{i}(t)\right\rangle=0,\left\langle\xi_{i}(t) \xi_{j}\left(t^{\prime}\right)\right\rangle=2 \delta_{i j} \delta\left(t-t^{\prime}\right)$.

The multiplier 2 in front of the Kronecker delta $\delta_{i j}$ makes it possible to unambiguously write down the Fokker-Planck equation and to ascribe the meaning of the diffusion coefficient to the function $N\left(h_{g}\right)$ [see Eq. (10) below] ${ }^{2}$. Expression (10) for $N\left(h_{g}\right)$ also takes the constants into account, which determine the fluctuation intensities for random quantities.

According to the regularities observed during SPD, the self-organization process in cellular structures, which results in the formation of new grain boundaries, takes a longer time than the formation of elementary dislocations does (here, a large difference between the main defects - dislocations and GBs is meant). In this connection, the establishment of a dynamic equilibrium in the system follows the evolution of the grain boundary density, so that we may use the adiabatic approximation, $\tau_{h_{g}} \gg \tau_{h_{D}}$. In the framework of the latter, we put $\tau_{h_{D}} \partial h_{D} / \partial t=0$ in Eq. (5) and obtain the Langevin stochastic differen-

${ }^{2}$ In this sense, the diffusion coefficient reflects the process of mutual penetration of structural defects belonging to different levels, which results in the self-organized equalizing of defect concentrations over the whole volume. 
tial equation (SDE) for the random variable $h_{g}{ }^{3}$,

$\tau_{h_{g}} \dot{h}_{g}=F\left(h_{g}\right)+\sqrt{N\left(h_{g}\right)} \xi(t)$.

The deterministic force $F\left(h_{g}\right)$ and the effective intensity of fluctuations $\sqrt{N\left(h_{g}\right)}$ of the random variable are formulated as follows [19]:

$$
\begin{aligned}
& F\left(h_{g}\right) \equiv \varphi_{0 g}+\frac{\varphi_{0 D} \varphi_{g D}}{\varphi_{1 \mathrm{D}}}+ \\
& +\left(\frac{\varphi_{g D}^{2}}{\varphi_{1 \mathrm{D}}}-2 \frac{\psi_{g D} \varphi_{0 D}}{\varphi_{1 \mathrm{D}}}-\varphi_{1 g}\right) h_{g}+ \\
& +\left(\varphi_{2 g}-3 \frac{\psi_{g D} \varphi_{g D}}{\varphi_{1 \mathrm{D}}}\right) h_{g}^{2}+\left(2 \frac{\psi_{g D}^{2}}{\varphi_{1 \mathrm{D}}}-\varphi_{3 g}\right) h_{g}^{3}, \\
& N\left(h_{g}\right) \equiv \frac{\left(\varphi_{g D}-2 \psi_{g D} h_{g}\right)^{2}}{\varphi_{1 \mathrm{D}}^{2}} N_{D}+N_{g} .
\end{aligned}
$$

When proving relation (10), which corresponds to the multiplicative noise, the dispersion properties of the independent Gaussian random variables [30] were taken into account. To avoid misunderstanding, a remark should be made that the direct transformations lead to the stochastic terms

$$
\left[\frac{\left(\varphi_{g D}-2 \psi_{g D} h_{g}\right)}{\varphi_{1 \mathrm{D}}} \sqrt{N_{D}}+\sqrt{N_{g}}\right] \xi(t),
$$

for which the square of their amplitudes is different from the effective noise intensity (10).

To develop the analysis further, the Langevin equation (8) has to be put in correspondence with a definite form of the Fokker-Planck equation, which describes the evolution of the distribution density function $p\left(h_{g}, t\right)$ for stochastic fluctuating variables (in our case, this is the parameter $h_{g}$ ). The following approaches are used most often when determining the solution: the calculus in the Itô interpretation (the $I$-form), the Stratonovich calculus (the $S$-form), and the kinetic form (the $K$-form) [30,31].

In the case of Itô calculus, the stochastic processes $h_{g}(t)$ and $d W(t)^{4}$ satisfy the criterion of the Markov

\footnotetext{
${ }^{3}$ Every implementation $\xi(t)$ of the random process is assumed to correspond to the formation of $h_{g}(t)$ of a new random process, i.e. the GB density acquires a random value at any time moment $t$.

4 This is a representation of the Wiener process for the stochastic differential equation (20) [30], which is a mathematical model of the Brownian motion for a random oscillation with the continuous time.
}

process and seem to be statistically independent [32], because the definition of the Itô integral reflects the absence of correlations between the random process $h_{g}(t)$ and the random force $W(t)$ at the time moment $t$. In the general case, the Itô form is used for solving the systems with discrete-time dynamics, which are mainly dealt with in biological systems (for example, to simulate the birth and death of living organisms) [28].

Integrating Eq. (8) with the help of the Stratonovich form makes it possible to automatically take the correlations between the random process $h_{g}(t)$ and the random variable $W(t)$ at short time intervals into account. For this purpose, the corresponding calculations should be performed at the intermediate points of the integration mesh,

$\sqrt{N\left(h_{g}\left(\frac{t_{i}+t_{i-1}}{2}\right)\right)} d W\left(t_{i}\right)$.

This procedure reflects the behavior of real physical systems with continuous time and memory $[1,33,34]$.

Hence, in this work, the Stratonovich approach was applied to the description of the fragmentation process in a metal polycrystalline structure under the action of SPD. This approach did not reveal qualitative changes in comparison with the Itô calculus.

The corresponding Fokker-Planck equation is written as follows [19]:

$$
\begin{aligned}
& \dot{p}\left(h_{g}, t\right)=-\frac{\partial}{\partial h_{g}} D^{(1)}\left(h_{g}\right) p\left(h_{g}, t\right)+ \\
& +\frac{\partial^{2}}{\partial h_{g}^{2}} D^{(2)}\left(h_{g}\right) p\left(h_{g}, t\right)
\end{aligned}
$$

where the functions

$D^{(1)}\left(h_{g}\right)=\frac{F\left(h_{g}\right)}{\tau_{h_{g}}}+\sqrt{\frac{N\left(h_{g}\right)}{\tau_{h_{g}}^{2}}} \frac{d \sqrt{N\left(h_{g}\right) / \tau_{h_{g}}^{2}}}{d h_{g}}$,

and

$D^{(2)}\left(h_{g}\right)=\frac{N\left(h_{g}\right)}{\tau_{h_{g}}^{2}}$

(or the Kramers-Moyal coefficients) [30] play the role of the drift and diffusion coefficients, respectively.

The stationary solutions (these are the probability density distributions for the realization of the states $h_{g}$ at $\left.\partial p\left(h_{g}, t\right) / \partial t=0\right)$ of Eqs. (8) and (13) look like $p\left(h_{g}\right)=Z^{-1} \exp \left(U_{\text {ef }}\left(h_{g}\right)\right)$.

ISSN 2071-0194. Ukr. J. Phys. 2019. Vol. 64, No. 6 
Here, the normalizing constant ${ }^{5}$

$$
Z=\int_{0}^{+\infty} \exp \left(U_{\mathrm{ef}}\left(\hat{h}_{g}\right)\right) d \hat{h}_{g}
$$

and the effective synergetic potential

$U_{\text {ef }}\left(h_{g}\right)=-\frac{1}{2} \ln \left(N\left(h_{g}\right)\right)+\tau_{h_{g}} \int_{0}^{h_{g}} \frac{F\left(\hat{h}_{g}\right)}{N\left(\hat{h}_{g}\right)} d \hat{h}_{g}$,

which evaluates the effective energy of the system, but does not have the physical sense of its internal energy, were introduced.

Let us derive an equation for the stationary values of the GB density $h_{g}$. It is determined from a necessary condition for the existence of extrema in distribution density (16) or effective potential (18): $d p\left(h_{g}\right) / d h_{g}=0$. The obtained maxima of the effective synergetic potential correspond to the maxima of the distribution density, which describe the formation of stable states (boundary structures), whereas the minima correspond to unstable realizations. Thus, the stationary condition brings about the expression

$\frac{d U_{\mathrm{ef}}\left(h_{g}\right)}{d h_{g}} \equiv F\left(h_{g}\right)-\frac{1}{2 \tau_{h_{g}}} \frac{d N\left(h_{g}\right)}{d h_{g}}=0$.

Its transformation makes it possible to obtain an equation that determines the positions of stationary states [19],

$$
\begin{aligned}
& \left(2 \frac{\psi_{g D}^{2}}{\varphi_{1 \mathrm{D}}}-\varphi_{3 g}\right) h_{g}^{3}+\left(\varphi_{2 g}-3 \frac{\psi_{g D} \varphi_{g D}}{\varphi_{1 \mathrm{D}}}\right) h_{g}^{2}+ \\
& +\left(\frac{\varphi_{g D}^{2}}{\varphi_{1 \mathrm{D}}}-2 \frac{\psi_{g D} \varphi_{0 D}}{\varphi_{1 \mathrm{D}}}-4 \frac{\psi_{g D}^{2}}{\tau_{h_{g}} \varphi_{1 \mathrm{D}}^{2}} N_{D}-\varphi_{1 g}\right) h_{g}+ \\
& +\varphi_{0 g}+\frac{\varphi_{0 D} \varphi_{g D}}{\varphi_{1 \mathrm{D}}}+2 \frac{\psi_{g D} \varphi_{g D}}{\tau_{h_{g}} \varphi_{1 \mathrm{D}}^{2}} N_{D}=0 .
\end{aligned}
$$

It is evident that the positions of the extrema in the effective synergetic potential (18), which separate the modes of metal fragmentation at SPD, do not depend on the noise intensity $N_{g}$.

Note also that expression (19) derived in the framework of the Stratonovich approach is somewhat different from its counterpart derived in the framework

\footnotetext{
${ }^{5}$ The integration limits are restricted by the physical interpretation of the parameter $h_{g}$.
}

of the Itô calculus. Namely, in the latter variant, the terms that involve the interaction with the fluctuations of $N_{D}$ (these are two terms in Eq. (19) that include the quantity $N_{D}$ as a multiplier) are additionally multiplied by 2 . Thus, the two-fold increase in the intensity of the dislocation density fluctuations, $2 N_{D}$, makes it possible to obtain results in the framework of the Stratonovich interpretation that are equivalent to those obtained in the Itô approach.

However, it is worth noting that the synergetic potential (18) and, accordingly, the distribution density $p\left(h_{g}\right)$ [Eq. (16)] acquire another form owing to the renormalization of the effective noise intensity $N\left(h_{g}\right)$. It is so because the latter differs from its counterpart in the Itô approach only in the first term (a constant of $1 / 2$ is available). This constant leads to the redistribution of the probability distribution density $p\left(h_{g}(t)\right)$ of the stochastic variable $h_{g}$, which obviously favors modifications in the character of time dependences of the GB density. In other words, the intensity of fluctuations of the parameter $h_{g}$ around the stationary system states changes. However, the formed stationary morphology of the material (a boundary structure with a constant value of $h_{g}$ ) remains unchanged irrespective of the calculus form choice.

In work [19], stationary solutions of the effective synergetic potential (18) and the formation of phase diagrams with the help of the Stratonovich calculus were studied. This approach of stochastic integration was used in this work as well, because its aim was not reduced to the study of only the formation of stationary SMC or NC structures, but it also included the study of the peculiarities in the evolution of $h_{g}$ during SPD.

\section{Procedure for Constructing the Time Dependences of GB Densities}

In order to analyze the kinetics of the GB density $h_{g}$ with regard for fluctuations of the basic parameters $N_{D, g}$, let us write down the Langevin equation with the multiplicative noise in the stochastic differential form. For this purpose, we should multiply Eq. (8) by $d t$. As a result, we obtain

$\tau_{h_{g}} d h_{g}=F\left(h_{g}\right) d t+\sqrt{N\left(h_{g}\right)} d W(t)$,

where $d W(t)=W(t+d t)-W(t) \equiv \xi(t) d t$ is a Wiener process with the white-noise properties [28, 30, 32]

$\langle d W(t)\rangle=0, \quad\left\langle(d W(t))^{2}\right\rangle=2 d t$. 
In this case, the noise is determined as the time derivative of the Wiener process, $\xi(t)=d W(t) / d t$.

Recall that the distribution of random fluctuations $\xi(t)$ over their $\xi$-values is the normal (Gaussian) distribution [30]

$P(\xi)=\frac{1}{\sigma \sqrt{2 \pi}} \exp \left\{-\frac{(\xi-\mu)^{2}}{2 \sigma^{2}}\right\}$

for which the first and second moments of the stochastic source are determined as follows:

$\mu \equiv\langle\xi(t)\rangle=0, \quad \sigma^{2} \equiv\left\langle\xi^{2}(t)\right\rangle=2 \delta(0)=2$.

It is worth noting that, in the framework of the examined case, the Gaussian white noise is considered as a limit of the real physical noise with a finite source intensity.

Let us rewrite Eq. (20) in a more general form,

$d h_{g}=D^{(1)}\left(h_{g}\right) d t+\sqrt{D^{(2)}\left(h_{g}\right)} d W(t)$.

Hence, in the framework of the Stratonovich approach (the $S$-form), the diffusion process is governed by the drift coefficient $D^{(1)}\left(h_{g}\right)$ [Eq. (14)] and the diffusion coefficient $D^{(2)}\left(h_{g}\right)$ [Eq. (15)] [30].

Note that the Stratonovich and Itô calculus forms are interrelated and allow a mutual transformation to be carried out $[28,32]$. If the initial $\operatorname{SDE}(20)$ is given in the Stratonovich interpretation, then, taking properties (21) into account, it is always possible to pass to the equivalent SDE in the framework of the Itô interpretation ${ }^{6}$ by subtracting the expression $g\left(h_{g}\right) d g\left(h_{g}\right) / d h_{g}$, where $g\left(h_{g}\right)=\sqrt{N\left(h_{g}\right) / \tau_{h_{g}}^{2}}$, from expression (14) for the drift coefficient. In turn, the backward transfromation is performed by adding the expression $g\left(h_{g}\right) d g\left(h_{g}\right) / d h_{g}$. In such a way, the initial and equivalent forms of SDE will have a single common solution.

Depending on the choice of the interpretation form for coefficients (14) and (15), the Langevin SDE (23) will be different in form and have a different physical meaning. It is evident that SDE in the Stratonovich form describes the diffusion process with a

\footnotetext{
6 Below, to make the consideration unambiguous, the calculus form of SDE, which is determined by the form of coefficients (14) and (15), is interpreted in accordance with the original interpretation irrespective of the stochastic process representation.
}

transfer, since the second term in the definition of the drift coefficient $(14), g\left(h_{g}\right) d g\left(h_{g}\right) / d h_{g}$, generates a noise-induced transition. This term is more commonly known in the literature as the "fault" transfer, since it does not enter the original phenomenological equation (8) or SDE (20) $[28,30]$. However, it is known that this term only results in physical consequences, because it simulates real systems with a medium, in which fast fluctuations take place; i.e. it involves the correlation between the random medium and the system.

It should be noted that if the kinetics of the system undergoes the action of an additive noise, i.e. if $N\left(h_{g}\right)=$ const in Eq. (20), there is no principal difference between the Itô and Stratonovich calculus systems. However, in the case of multiplicative noise, i.e. if $N\left(h_{g}\right) \neq$ const, and if the effect of random force depends on the system state, the correlation contained in the Stratonovich integral leads to a systematic contribution to the evolution of the random process $h_{g}(t)$. Hence, the SDE in the Stratonovich form is more appropriate for the description of a real physical situation associated with rapid changes in the medium $[28,30]$. However, it is worth noting that there are no objective reasons that would give the unambiguous preference to a definite SDE interpretation. In any case, a decisive criterion for the choice to be correct is the correspondence of analytically found results to experimental data.

In particular, the correctness of a theoretical consideration dealing with the behavior of the main variables of a physical system (in our case, this is $h_{g}$ ) is usually confirmed by comparing the results of numerical simulations for a number of SDE forms. Despite a wide spectrum of available calculus forms, the Stratonovich approach, in which the diffusion process is taken into account, and the Itô form are considered to be the most justified.

The numerical solution of relation (23) can be found making use of the Euler method [20, 22]. By applying the discrete approximation to the differential of the random variable, $d W(t)=\sqrt{\Delta t} W_{i}$, we obtain a standard iterative procedure for integrating (solving) Eq. (23):

$h_{g_{i+1}}=h_{g_{i}}+D^{(1)}\left(h_{g_{i}}\right) \triangle t+\sqrt{D^{(2)}\left(h_{g_{i}}\right) \triangle t} W_{i}$.

Using coefficients (14), (15) and expressions (9), (10), let us calculate the time dependence of the GB density $h_{g}$. In particular, in the case of the Stratonovich

ISSN 2071-0194. Ukr. J. Phys. 2019. Vol. 64, No. 6 
calculus, the iterative procedure has the following explicit form:

$$
\begin{aligned}
& h_{g_{i+1}}=h_{g_{i}}+ \\
& +\left[\frac{F\left(h_{g_{i}}\right)}{\tau_{h_{g}}}+2 \frac{\left(2 \psi_{g D}^{2} h_{g_{i}}+\varphi_{g D} \psi_{g D}\right)}{\tau_{h_{g}}^{2} \varphi_{1 \mathrm{D}}^{2}} N_{D}\right] \Delta t+ \\
& +\sqrt{\frac{N\left(h_{g_{i}}\right)}{\tau_{h_{g}}^{2}} \Delta t} W_{i} .
\end{aligned}
$$

The solution of SDE (23) was determined in the time interval $t \in[0, T]$ for a definite number of iterations $N$ (the number of points in the time dependence). Accordingly, the time increment was determined by the expression $\Delta t=T / N$. The force $W_{i}$ has the following characteristics:

$$
\left\langle W_{i}\right\rangle=0, \quad\left\langle W_{i} W_{i^{\prime}}\right\rangle=0, \quad\left\langle W_{i}^{2}\right\rangle=2,
$$

which corresponded to the white noise moments (21).

The simulation of a random force with the properties of a white noise was carried out using the BoxMuller model [35],

$W_{i}=\sigma \sqrt{-2 \ln r_{1}} \cos \left(2 \pi r_{2}\right), \quad r_{1,2} \in(0,1]$,

where, according to the second moment in Eq. (26), the dispersion $\sigma=\sqrt{2}$, and $W_{i}$ is a completely random number, whose properties are described by Eqs. (26) and (22). The pseudorandom numbers $r_{1}$ and $r_{2}$ have a uniform distribution and were repeated after a certain period.

\section{Self-Similar Fragmentation Mode}

Let us determine conditions for a self-similar behavior to appear in the proposed two-defect system. This behavior is obtained by differentiating the multidimensional thermodynamic potential (1) (or the density of the effective internal energy). The corresponding uniform distribution function, which is typical of self-similar systems, is defined by the dependence

$P_{q}(y)=y^{-q} \Pi\left(h_{g}\right), \quad y=h_{g} h_{g}^{s}$,

where $q$ is the uniformity order or the power exponent of distribution [9], which determines the slope angle of the linear plot. Note that the power exponent can acquire both integer and fractional values. In particular, the mode of self-organized criticality is formed for the power exponent $2 q=1.5$ [36].
Let us analyze the stationary distribution of the density $h_{g}$ [Eq. (16)], which is determined by the effective synergetic potential (18). If the condition $N_{g} \gg N_{D}$ is satisfied, then, at $N_{D}=0$, the effective noise intensity equals $N\left(h_{g}\right) \equiv N_{g}$ [see definition (10)]. Accordingly, the distribution density is determined by the formula

$p\left(h_{g}\right)=Z^{-1} N_{g}^{-1 / 2} \exp \left(\frac{\tau_{h_{g}}}{N_{g}} \int_{0}^{h_{g}} F\left(\hat{h}_{g}\right) d \hat{h}_{g}\right)$.

It is evident that expression (29) differs substantially from the uniform distribution function (28).

The fluctuations of the dislocation density under the condition

$N_{D} \gg N_{g}$

- in this case, we may put $N_{g}=0$ in Eq. (10) bring about the following expression for the effective synergetic potential:

$U_{\mathrm{ef}}\left(h_{g}\right)=\ln \left(\frac{\left(\varphi_{g D}-2 \psi_{g D} h_{g}\right)^{2}}{\varphi_{1 \mathrm{D}}^{2}} N_{D}\right)^{-1 / 2}+$
$+\tau_{h_{g}} N_{D}^{-1} \varphi_{1 \mathrm{D}}^{2} \int_{0}^{h_{g}} \frac{F\left(\hat{h}_{g}\right)}{\left(\varphi_{g D}-2 \psi_{g D} \hat{h}_{g}\right)^{2}} d \hat{h}_{g}$.

In turn, the distribution density reads

$p\left(h_{g}\right)=Z^{-1}\left(\varphi_{g D}-2 \psi_{g D} h_{g}\right)^{-1} \varphi_{1 \mathrm{D}} N_{D}^{-1 / 2} \times$
$\times \exp \left(\frac{\tau_{h_{g}} \varphi_{1 \mathrm{D}}^{2}}{N_{D}} \int_{0}^{h_{g}} \frac{F\left(\hat{h}_{g}\right)}{\left(\varphi_{g D}-2 \psi_{g D} \hat{h}_{g}\right)^{2}} d \hat{h}_{g}\right)$.

It is evident that the resulting distribution is characterized by the power-law asymptotics $p\left(h_{g}\right) \propto h_{g}^{-1}$ at $\varphi_{g D} \rightarrow 0$ and in the interval $0<h_{g} \leq h_{g}^{\max }$. Then Eq. (32) is reduced to the canonical form (28)

$P_{q}(y)=y^{-1} \Pi\left(h_{g}\right), \quad y=h_{g} h_{g}^{s}$,

where

$\Pi\left(h_{g}\right)=Z^{-1}(-2)^{-1} \psi_{g D}^{-1} \varphi_{1 \mathrm{D}} N_{D}^{-1 / 2} \times$

$\times \exp \left(\frac{\tau_{h_{g}} \varphi_{1 \mathrm{D}}^{2}}{N_{D}} \int_{0}^{h_{g}} \frac{F\left(\hat{h}_{g}\right)}{4 \psi_{g D}^{2} \hat{h}_{g}^{2}} d \hat{h}_{g}\right)$. 


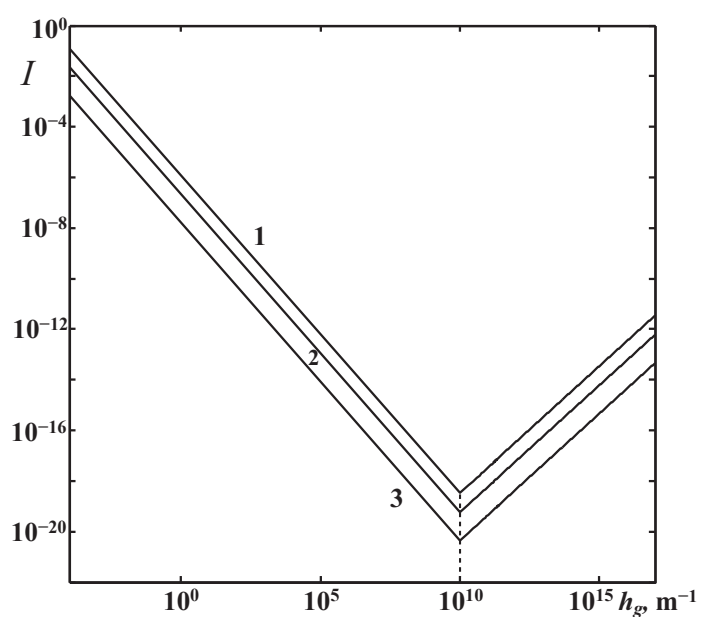

Fig. 1. Integrand (35) plotted for the parameters values $\varepsilon_{i i}=-0.1 \%, I_{2}=10^{-4 \%}$, and $N_{D}=2(1), 10$ (2), and $100 \mathrm{~J}^{2} \mathrm{~s} \mathrm{~m}^{-2}(3)$

Hence, distribution (33) is uniform, if the function $\Pi\left(h_{g}\right)$ [Eq. (34)] acquires a constant value, i.e. $\Pi\left(h_{g}\right)=$ const.

Let us determine conditions for the formation of a self-similar distribution. For this purpose, let us analyze the subintegral expression in Eq. (34),

$$
\begin{aligned}
& I=\frac{\tau_{h_{g}} \varphi_{1 \mathrm{D}}^{2}}{N_{D}}\left[\frac{\varphi_{0 g}}{4 \psi_{g D}^{2} h_{g}^{2}}-\left(\frac{\varphi_{0 D}}{2 \varphi_{1 \mathrm{D}} \psi_{g D}}+\frac{\varphi_{1 g}}{4 \psi_{g D}^{2}}\right) \frac{1}{h_{g}}+\right. \\
& \left.+\frac{\varphi_{2 g}}{4 \psi_{g D}^{2}}+\left(\frac{1}{2 \varphi_{1 \mathrm{D}}}-\frac{\varphi_{3 g}}{4 \psi_{g D}^{2}}\right) h_{g}\right] .
\end{aligned}
$$

From this result, one can see that the contributions of the first and second terms decrease as $h_{g}$ grows, whereas the third term gives a small contribution at $\varphi_{2 g} \approx 0 \mathrm{~J}$. In particular, the influence of those terms can be considerably reduced by increasing the value of the parameter $\psi_{g D}$. However, as the GB density $h_{g}$ grows, the fourth term gives a significant contribution to the distribution function (33), because it changes the distribution character: the transition from the power-law dependence to the exponential one takes place. From this viewpoint, the power-law distribution will be realized only at $\varphi_{1 \mathrm{D}} \ll 1 \mathrm{~J} \mathrm{~m}$ or $N_{D} \gg 1 \mathrm{~J}^{2} \mathrm{~s} \mathrm{~m}^{-2}$ and in the limited interval of GB densities $0<h_{g} \leq h_{g}^{\max }$.

The direct physical meaning of the indicated selfsimilarity conditions can be understood more clearly from expression (10), because, under such conditions, the effective intensity of the noise increases and, accordingly, the spread of the values of the GB density $h_{g}$ increases. However, it should be noted that the order parameter $h_{g}$ cannot be infinitely large, because, from the physical viewpoint, a fragmented metallic specimen corresponds to an amorphous structure under such conditions, and this is practically impossible, if only the known SPD methods are applied. Thus, a quasifractal structure with characteristic grain size is formed only in a limited interval. At a high GB density $h_{g}$, the distribution rapidly decreases and becomes exponential. Accordingly, it is assumed that smaller grains are not formed in the cellular structure of a metal.

In Fig. 1, function (35), i.e. the integrand in Eq. (34), is plotted. According to this figure, the integral in expression (34) is the smallest at $h_{g}<$ $<10^{10} \mathrm{~m}^{-1}$. Above this value, the contribution of the function $\Pi\left(h_{g}\right)$ [Eq. (34)] to the distribution begins to increase gradually. Accordingly, the integral gives a main contribution to the resulting distribution function (33), which is accompanied by an exponential reduction (see Fig. 2). Thus, a necessary condition for self-similarity is the power-law character of the distribution function, because the reduction rate of such a dependence is much lower in comparison with that of the exponential one.

Hence, the power-law distribution, which is characteristic of the self-similar behavior, really exists in a limited interval of the GB density values. When $h_{g}$ exceeds a certain critical value $h_{g}^{\max }$, the system loses its self-similar properties. Note that distribution (33) was obtained in the framework of the Stratonovich calculus. In the case of Itô calculus, the power exponent of the distribution acquires a value of -2 . However, as was mentioned above, the choice of a calculus has only a quantitative effect on the behavior of the system, which reveals itself in the renormalization of the noise intensities $N_{D}$ and $N_{g}$.

Note also that if the necessary conditions for the self-similar behavior are satisfied $\left(\varphi_{g D}=0 \mathrm{~J}\right.$ and $\varphi_{1 \mathrm{D}} \ll 1 \mathrm{~J} \mathrm{~m}$ or $\left.N_{D} \gg 1 \mathrm{~J}^{2} \mathrm{~s} \mathrm{~m}^{-2}\right)$, the phase diagram, which determines fragmentation modes [19], makes no sense. This conclusion follows from the fact that, at the indicated parameter values, only those terms make substantial contributions that involve the interaction with the fluctuations of $N_{D}$. These are the terms with the multiplier $N_{D}$ in Eq. (19). In this case, according to Eq. (33) with $h_{g}=0 \mathrm{~m}^{-1}$, the dis- 
tribution diverges, because the integral in the function $\Pi\left(h_{g}\right)$ [Eq. (34)] does not contribute. With the growth of $h_{g}$, the distribution remains to be powerlaw until the contribution of the last term in Eq. (35) becomes substantial and distribution (33) acquires an exponentially decaying character. Hence, in the selected interval, the system has a single maximum of the distribution function at the point $h_{g} \approx 0 \mathrm{~m}^{-1}$; then, up to $h_{g}=h_{g}^{\max }$, the distribution is power-law; and at $h_{g}>h_{g}^{\max }$, it begins to exponentially decay (see Fig. 2). In the latter case, the probability of the formation of GB densities $h_{g}>h_{g}^{\max }$ is small, but different from zero.

Let us consider the dependences $P\left(h_{g}\right)(33)$, which are plotted in Fig. 2. Note that the results obtained are numerically normalized in the given interval of $h_{g}$-values. In the general case, the calculation of the analytic normalizing constant is impossible, because the distribution diverges at $h_{g} \rightarrow 0 \mathrm{~m}^{-1}$. All the curves in Fig. 2 were plotted on the loglog scale, which allows one to observe the formation of a power-law dependence at $h_{g}<10^{10} \mathrm{~m}^{-1}$ and, accordingly, the realization of the self-similar mode. As one can see, the value $h_{g} \approx 10^{10} \mathrm{~m}^{-1}$ determined from Fig. 1 corresponds to the exhibited dependences, although the distribution preserves visually its tendency for some time. The plotted dependences testify that, as the noise intensity $N_{D}$ increases, the interval of GB density values, at which the distribution function $P\left(h_{g}\right)$ becomes uniform, grows (see the inset). In particular, the slope of the linear sections is constant irrespective of the model noise parameters. Hence, if condition (30) is satisfied, a self-similar behavior is established, which is determined by the absence of a characteristic scale for the GB density in the interval $h_{g}<10^{10} \mathrm{~m}^{-1}$. However, in order to obtain statistical parameters for the time dependences of $h_{g}$, a more detailed multifractal fluctuation analysis [37] is required.

Let us check the linearity of the dependences depicted in Fig. 2 in the indicated limits $0<h_{g} \leq$ $\leq 10^{10} \mathrm{~m}^{-1}$. For this purpose, let us apply the correlation analysis $[35,38]$. Doing in such a way and applying the least squares method in the interval $10^{-5} \mathrm{~m}^{-1} \leq h_{g} \leq 10^{10} \mathrm{~m}^{-1}$, we obtain the regression equation

$\lg \left(P\left(h_{g}\right)\right)=A \lg \left(h_{g}\right)+B$.

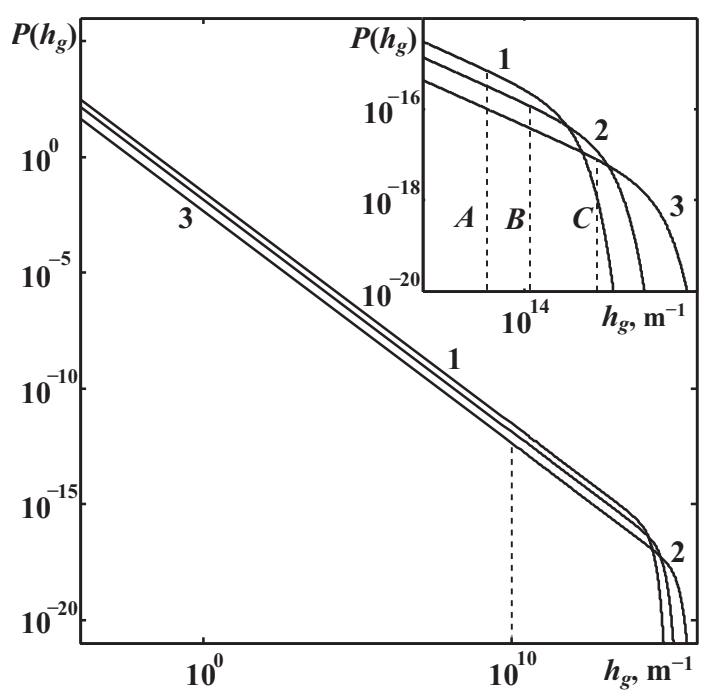

Fig. 2. Distribution function (33) plotted for the parameters values $\varphi_{g D}=0, \varepsilon_{i i}=-0.1 \%, I_{2}=10^{-4 \%}, N_{g}=0$, and $N_{D}=2(1), 10(2)$, and $100 \mathrm{~J}^{2} \mathrm{~s} \mathrm{~m}^{-2}$ (3)

The quality of the regression model is determined in a standard manner with the help of the coefficient of determination $R^{2}$, which is calculated as follows:

$R^{2}=1-\frac{\sum_{i=1}^{n}\left(y_{i}-\hat{y}\right)^{2}}{\sum_{i=1}^{n}\left(y_{i}-\bar{y}\right)^{2}}$,

where $y \equiv \lg \left(P\left(h_{g}\right)\right)$, $\hat{y}$ are the values corresponding to Eq. (36), $\bar{y}=\sum_{i=1}^{n} y_{i} / n$ is the mean value, and the values $y_{i}$ are determined from expression (33). The number of points in the indicated interval $10^{-5} \mathrm{~m}^{-1} \leq h_{g} \leq 10^{10} \mathrm{~m}^{-1}$ was taken to be equal to $n=10^{5}$.

The values of the coefficients $A, B$, and $R^{2}$ are quoted in Table 2 for each case. One can see that the values of the coefficient $A$ obtained for the curves in Fig. 2 approximately equal to -1 , which agrees with the power-law form of expression (equal $A \approx-1$ )

Table 2. Parameters of linear regression

(36) and the coefficients of determination (37) for the curves depicted in Fig. 2

\begin{tabular}{|c|c|c|c|}
\hline $\begin{array}{l}\text { Curve } \\
\text { number }\end{array}$ & $A$ & $B$ & $R^{2}$ \\
\hline 1 & -1.032 & -2.533 & 0.9992 \\
2 & -1.006 & -4.087 & 0.9995 \\
3 & -1.001 & -5.407 & 0.9995 \\
\hline
\end{tabular}



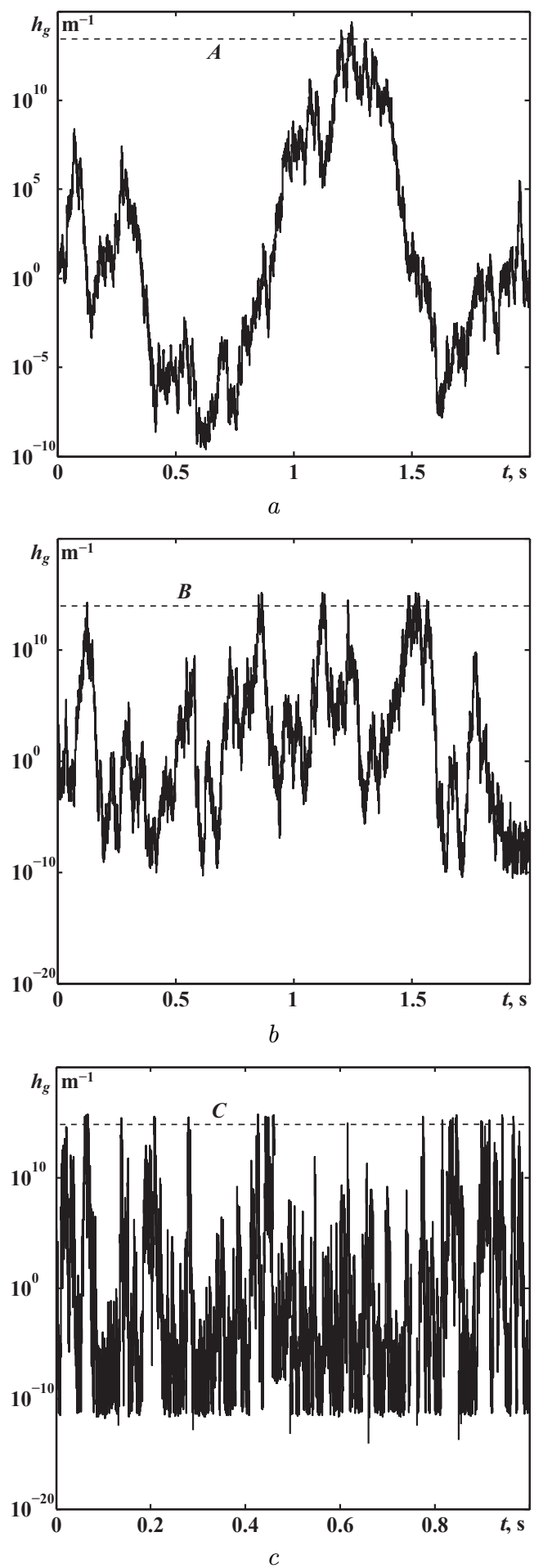

Fig. 3. Time dependences of $h_{g}$ for the parameters values $\varphi_{g D}=0, \varepsilon_{i i}=-0.1 \%, I_{2}=10^{-4 \%}, N_{g}=0$, and $N_{D}=2(a)$, $10(b)$, and $100 \mathrm{~J}^{2} \mathrm{~s} \mathrm{~m}^{-2}(c)$ provided the integer value $q=1$ of the power exponent. The corresponding correlation coefficients $R^{2}$ [Eq. (37)], which are also quoted in Table 2, testify to a high correlation between the regression equation (36) and expression (33) in the corresponding linear sections. Therefore, distribution (33) is really a power-law one. Furthermore, as was already mentioned, the growth of the noise intensity $N_{D}$ increases the lengths of linear $h_{g}$-sections (see the boundaries of linear sections marked with dashed lines in the inset). Hence, the limiting size of grains in a quasifractal metal structure formed during SPD will permanently decrease under the established conditions.

Figure 3 demonstrates the time dependences of $h_{g}$, which correspond to the parameters of curves $1-3$ in Fig. 2 and were calculated following the method described in Section 4. The evolution of the GB density values is exhibited on the logarithmic scale, which makes it possible to demonstrate the self-similar behavior of the order parameter of the system in a certain limited interval. In particular, one can see that this behavior reveals itself at the expense of a drastic increase of the parameter $h_{g}$ on various scales. From the obtained results, it is evident that the increase of the intensity of the dislocation density fluctuations $N_{D}$ gives rise to the realization of a self-similar behavior in a larger range of scales, which is confirmed by the extent of the power-law distribution in Fig. 2. The dashed curves correspond to the maximum permissible values for the maximum of the parameter $h_{g}$, below which the self-similar behavior is observed (see the inset in Fig. 2). In addition, the analysis of time dependences shows that the power-law form of the distribution function $P\left(h_{g}\right)$ [Eq. (33)] is restricted not only by the maximum, but also the minimum value of the GB density, which decreases with the growth of the noise intensity $N_{D}$.

Hence, the self-similar character of the metal structure evolution is realized by activating the self-organized processes of various kinds, which arise as a result of the noise inherent to main parameters. It is known that it is the interaction of structural defects with one another and with defects of other structural levels-in our case, this is the interaction of GBs with dislocations, other boundaries, and structural inhomogeneities, such as structural defects of other levels, thermal fluctuations, thermodynamical phases of the substance, impurities, vacancies, and so forth-that leads to the manifestation of internal fluctuations and 
the changes in grain misorientations in the granular structure of a metal specimen $[1,27,29,39,40]$. As a result, the emerged boundary structure contains crystallites with a specific structure. Namely, according to the results of X-ray studies, those crystallites are fragmented into separate regions of coherent scattering (cells, subgrains) [1, 41-43].

\section{Conclusions}

On the basis of nonequilibrium evolution thermodynamics, the fragmentation process in a metal structure under the influence of SPD has been studied. As a result, the integrated picture of the basic modes for the ordinary and severe plasticities is obtained. The simulation of the defect formation processes is carried out in the framework of the two-defect model and making allowance for noise. Grain boundaries and dislocations were considered as the main structural defects, since the dislocations play a significant role in the formation of a fine grain structure and determine the plastic flow limit. A modification of the powerseries expansion of the internal energy density allowed the self-consistent behavior of structural defects during the formation of SMC or NC boundary structures to be described more accurately. The fluctuations of main parameters were assumed to reflect the stochastic interaction with other, unaccounted structural inhomogeneities (thermodynamic phases of the substance, impurities, inclusions, vacancies, structural defects of other levels, thermal fluctuations, and others), which are always available in a real metal structure. It is the result of such interactions in the course of SPD treatment that is responsible for the competition and transitions among different structural states (phases).

It is shown that the additive noise of the lower level demonstrates a multiplicative character at the upper, macroscopic level, which results in non-equilibrium transitions and the formation of new states in the system. A comparison of the Itô and Stratonovich calculus forms is carried out. In particular, it is shown that the change of the calculus gives rise only to the renormalization of the probability density for the stochastic variable distribution (realizations of the GB density). This renormalization manifests itself only in the character of the time dependences of the GB density owing to the changes in the intensity of GB density fluctuations around stable configurations and does not result in the changes of its stationary values. It is found that, for the given formulation of the problem, it is the Stratonovich approach that allows the actual fragmentation process to be described more adequately, because it involves rapid changes in the medium, as well as the prehistory of the crystalline structure development.

The conditions required for the formation of selfsimilar structures are analyzed. It is found that, under definite conditions, a self-similar behavior in a fragmented metallic specimen can be observed. It is found that, in the limited interval of order parameter values, $h_{g} \sim\left(10^{-5} \div 10^{10}\right) \mathrm{m}^{-1}$, the distribution function of the GB density acquires a power-law form. In particular, the growth in the intensity of stochastic source fluctuations results in a larger extension of the power-law distribution. As a result, there arises a quasifractal structure in a metal or alloy in a wider range of scales of the parameter $h_{g}$. Under the indicated conditions, the maximum grain size permanently decreases.

The author expresses his gratitude to the Ministry of Education and Science of Ukraine for the financial support of the work (project No.0118U003584 "Atomistic and statistical representation of formation and friction of nanodimensional systems").

1. R.Z. Valiev, I.V. Alexandrov. Bulk Nanostructured Metallic Materials: Production, Structure, and Properties (Akademkniga, 2007) (in Russian).

2. R.M. Kronover. Fractals and Chaos in Dynamic Systems. Fundamentals of Theory (Postmarket, 2000) (in Russian).

3. Ya.E. Beigelzimer, V.N. Varyukhin, D.V. Orlov, S.G. Synkov. Screw Extrusion-Process of Deformation Accumulation (TEAN, 2003) (in Russian).

4. A. Carpinteri, A. Spagnoli, S. Vantadori. A multifractal analysis of fatigue crack growth and its application to concrete. Engng. Fract. Mech. 77, 974 (2010).

5. L. Molenta, A. Spagnoli, A. Carpinteri, Rh. Jones. Fractals and the lead crack airframe lifing framework. Proc. Struct. Integr. 2, 3081 (2016).

6. V.S. Ivanova, A.A. Oksogoev. On the relationship of plastic deformation processes with a fractal structure corresponding to the change in the deformation scale level. Fiz. Mezomekh. 9, 17 (2006) (in Russian).

7. A.A. Ivanova, V.V. Lepov, V.S. Achikasova, A.M. Ivanov. Application of the statistical fractal concept in the analysis of the specimen deformation surfaces. Nauka Obrazov. No. 4, 89 (2016) (in Russian). 
8. V.A. Oborin, M.V. Bannikov, Y.V. Bayandin, M.A. Sokovikov, D.A. Bilalov, O.B. Naimark. Fractal analysis of fracture surface of aluminum alloy AMg6 under fatigue and dynamic loading. PNRPU Mech. Bull. No. 2, 116 (2015).

9. D.J. Amit. Field Theory, the Renormalization Group, and Critical Phenomena (McGraw-Hill, 1978).

10. O.I. Olemskoi, O.V. Yushchenko, T.I. Zhylenko. Study of conditions for hierarchical condensation near the phase equilibrium. Ukr. J. Phys. 56, 474 (2011).

11. L.S. Metlov. Nonequilibrium Evolution Thermodynamics and Its Applications (Noulidge, 2014) (in Russian).

12. L.S. Metlov. Nonequilibrium dynamics of a two-defect system under severe load. Phys. Rev. E. 90, 022124 (2014).

13. A.V. Khomenko, D.S. Troshchenko, L.S. Metlov. Thermodynamics and kinetics of solids fragmentation at severe plastic deformation. Condens. Matter Phys. 18, 33004 (2015).

14. A.V. Khomenko, D.S. Troshchenko, L.S. Metlov. Modelling of kinetics of modes of a fragmentation of materials at a severe plastic deformation. Metallofiz. Noveish. Tekhnol. 39, 265 (2017) (in Russian).

15. I.A. Lyashenko, A.V. Khomenko, L.S. Metlov. Thermodynamics and kinetics of boundary friction. Tribol. Int. 44, 476 (2011).

16. D.S. Troshchenko. Non-Equilibrium Evolution Thermodynamics of Metal Fragmentation with Account of Stochasticity. Ph.D. thesis (Sumy State Unuversity, 2018) (in Ukrainian).

17. A.D. Pogrebnyak, A.A. Bagdasaryan, I.V. Yakushchenko, V.M. Beresnev. The structure and properties of highentropy alloys and nitride coatings based on them. Russ. Chem. Rev. 83, 1027 (2014).

18. A.A. Goncharov, A.N. Yunda, R.Yu. Bondarenko, S.A. Goncharova. Modelling of thermal processes in the cutting insert with a protective coating. In Proceedings of the 2016 International Conference on Nanomaterials: Application and Properties (NAP-2016) (Sumy State University, 2016), Vol. 5, p. 02NEA06.

19. O.V. Khomenko, D.S. Troshchenko, Ya.O. Kravchenko, M.O. Khomenko. Additive gaussian noise effect on phase diagram of metal's fragmentation modes during severe plastic deformation. J. Nano- Electron. Phys. 9, 03045 (2017) (in Ukrainian).

20. A.V. Khomenko, Ya.A. Lyashenko. Periodic intermittent regime of a boundary flow. Tech. Phys. 55, 26 (2010).

21. A.V. Khomenko. Noise influence on solid-liquid transition of ultrathin lubricant film. Phys. Lett. A 329, 140 (2004).

22. A.V. Khomenko, I.A. Lyashenko. Phase dynamics and kinetics of thin lubricant film driven by correlated temperature fluctuations. Fluct. Noise Lett. 7, L111 (2007).
23. A.M. Glezer, I.E. Permyakova. Melt-Quenched Nanocrystals (CRC Press, 2013).

24. A.M. Glezer, L.S. Metlov. Physics of megaplastic (severe) deformation in solids. Phys. Solid State 52, 1162 (2010).

25. O.V. Khomenko, Ya.O. Lyashenko. Phase dynamics of a thin lubricant film between solid surfaces at the deformation defect of shear modulus. J. Phys. Stud. 11, 268 (2007) (in Ukrainian).

26. M.M. Protodyakonov, R.I. Teder, E.I. Ilnitskaya, O.P. Yakobashvili, I.B. Safronova, A.I. Tsykin, I.O. Kvashnina, N.N. Pavlova, L.N. Levushkin, Yu.V. Zefirov, A.A. Savelyev, M.O. Dolgova. The Distribution and Correlation of Physical Properties of the Rocks: A Reference Guide (Nedra, 1981) (in Russian).

27. E.V. Kozlov, N.A. Popova, N.A. Koneva. Regularities in plastic deformation of ultrafine-grained metallic materials. Deform. Razrush. Mater. No. 5, 2 (2014) (in Russian).

28. W. Horsthemke, R. Lefever. Noise-Induced Transitions. Theory and Applications in Physics, Chemistry, and Biology (Springer, 1984).

29. G.A. Salishchev, S.Yu. Mironov, S.V. Zherebtsov, A.N. Belyakov. Influence of plastic deformation on the change of boundary misorientation in metallic materials. Fiz. Mekh. Mater. 25, 42 (2016) (in Russian).

30. H. Risken. The Fokker-Planck Equation. Methods of Solution and Applications (Springer, 1989).

31. Yu. L. Klimontovich. Nonlinear Brownian motion. Phys.Usp. 37, 737 (1994).

32. C.W. Gardiner. Handbook of Stochastic Methods for Physics, Chemistry and the Natural Sciences (Springer, 1983).

33. Shuyong Jiang, Yanqiu Zhang, Lihong Zhao, Yufeng Zheng. Influence of annealing on NiTi shape memory alloy subjected to severe plastic deformation. Intermetallics 32, 344 (2013).

34. Wen Ma, Bin Chen, Fu-Shun Liu, Qing Xu. Phase transformation behaviors and mechanical properties of $\mathrm{Ti}_{50} \mathrm{Ni}_{49} \mathrm{Fe}_{1}$ alloy with severe plastic deformation. Rare Metals 32, 448 (2013).

35. W.H. Press, S.A. Teukolsky, W.T. Vetterling, B.P. Flannery. Numerical Recipes: The Art of Scientific Computing (Cambridge Univ. Press, 2007).

36. A.I. Olemskoi, A.V. Khomenko, D.O. Kharchenko. Selforganized criticality within fractional Lorenz scheme. Physica A 323, 263 (2003).

37. A.V. Khomenko, I.A. Lyashenko, V.N. Borisyuk. Selfsimilar phase dynamics of boundary friction. Ukr. J. Phys. 54, 1139 (2009).

38. A.M. Nazarenko. Econometrics (Sumy State Univ., 2003) (in Russian).

39. V.V. Malashenko. Collective overcoming of point defects by dislocations in the dynamic region. Fiz. Tverd. Tela 56, 1528 (2014) (in Russian).

ISSN 2071-0194. Ukr. J. Phys. 2019. Vol. 64, No. 6 
40. N.V. Prodanov, A.V. Khomenko. Computational investigation of the temperature influence on the cleavage of a graphite surface. Surf. Sci. 604, 730 (2010).

41. A.I. Bazhin, A.A. Goncharov, A.D. Pogrebnyak, V.A. Stupak, S.A. Goncharova. Superhardness effect in transitionmetal diborides films. Phys. Met. Metall. 117, 594 (2016).

42. A.V. Khomenko, N.V. Prodanov. Study of friction of Ag and Ni nanoparticles: an atomistic approach. J. Phys. Chem. C 114, 19958 (2010).

43. G.A. Malygin. Kinetic mechanism of the formation of fragmented dislocation structures upon large plastic deformations. Phys. Solid State 44, 2072 (2002).

Received 17.03.19. Translated from Ukrainian by O.I. Voitenko
О.В. Хоменко

САМОПОДІБНИЙ РЕЖИМ

ФРАГМЕНТАЦІЇ МЕТАЛІВ ПРИ ІНТЕНСИВНІЙ ПЛАСТИЧНІЙ ДЕФОРМАЦІІ

$\mathrm{P}$ е $з$ ю м е

В рамках нерівноважної еволюційної термодинаміки проведено подальше дослідження впливу адитивних флуктуацій на кінетику структурних дефектів при інтенсивній пластичній деформації, що являє новий метод опису режимів фрагментації та відповідних процесів самоорганізації. Встановлено, що у фрагментованому металевому зразку спостерігається самоподібна поведінка, при якій утворюється множина граничних структур із різними розмірами зерен. Такий режим реалізується за умови, що розподіл ймовірності реалізації значень густини меж зерен має степеневий вид. Порівняння отриманих результатів у формах Іто та Стратоновича продемонструвало відсутність якісних змін у поведінці системи. 